\title{
A Search for Long-Period Variables in Globular Clusters: M22 and IC4499
}

\author{
A. Sahay ${ }^{1}$, T. Lebzelter ${ }^{2,4}$ and P. R. Wood ${ }^{3}$ \\ ${ }^{1}$ Department of Applied Physics, Indian School of Mines, Dhanbad, India \\ ${ }^{2}$ University of Vienna, Department of Astrophysics, Türkenschanzstrasse 17, A1180 Vienna, Austria \\ ${ }^{3}$ Research School for Astronomy and Astrophysics, Australian National University, Canberra, Australia \\ ${ }^{4}$ Email: thomas.lebzelter@univie.ac.at
}

(RECEIVED November 22, 2013; ACCEPTED January 11, 2014)

\begin{abstract}
We report on the results of a long time photometric monitoring of the two metal-poor Galactic globular clusters, M22 and IC4499, searching for long-period variables (LPVs) on the upper giant branch. We detected 22 new LPVs in the field of M22 and confirmed the variability of six known variables. Periods could be determined for 16 of them. In the field of IC4499 we detected and characterised two new LPVs. Cluster membership is evaluated for all the variables based on photometry and literature data, and the location of the stars in $\log P$ - $K$-diagram is discussed. Our findings give further support to the presence of LPVs at metallicities as low as $[\mathrm{Fe} / \mathrm{H}]=-1.7$. The luminosity range where LPVs are found in metal-poor clusters is lower than in more metal-rich clusters.
\end{abstract}

Keywords: globular clusters: individual (IC4499) - globular clusters: individual (M22) - stars: AGB - stars: variables

\section{INTRODUCTION}

The Asymptotic Giant Branch (AGB) phase is a key stage in the stellar evolution of low- and intermediate-mass stars. It is characterised by nucleosynthesis, mixing, and mass-loss events, and it is therefore of fundamental importance for the cosmic matter cycle. The atmospheres of these stars are highly extended - not least due to the levitation of material by stellar radial pulsation - and this presents major challenge for determining the parameters of these objects (Lebzelter et al., 2010). A study of the variability of AGB stars provides another mechanism for determining the parameters of red giant stars, particularly their mass, their cumulative amount of mass loss, and possibly gross abundance variations such as a high helium content.

An interesting possibility is to study AGB stars in single stellar populations where metallicity, mass (age), and distance can be determined from other objects in that system. Therefore, two of us (TL and PW) have started a long time photometric monitoring programme of several Galactic and Magellanic Clouds globular clusters to get an extensive repository of AGB stars in these systems and to use the detected sample in each cluster to further investigate the properties of low- and intermediate-mass stars during this final evolutionary stage. The results on several of the clusters studied have been published already: NGC 104 (Lebzelter \& Wood, 2005), NGC 1846 (Lebzelter \& Wood, 2007), NGC 1978 and NGC 419 (Kamath et al., 2010), and NGC 362 and NGC 2808 (Lebzelter \& Wood, 2011). Here we present results on two more clusters, namely M22 (NGC 6656) and IC4499.

M22 $(l=9.9 \mathrm{deg}, b=-7.6 \mathrm{deg})$ is a galactic globular cluster at a distance of $\sim 3.1 \mathrm{kpc}$ from the sun and a mean metallicity of $[\mathrm{Fe} / \mathrm{H}]=-1.64$ (Harris, 1996). An age estimate of 12.5 Gyr (van den Berg et al., 2013) places it among the bulk of the Galactic globular clusters. An average reddening value of $E(B-V)=0.38$ has been found with significant variations across the cluster (Kunder et al., 2013). It was noticed quite early (Hesser, Hartwick, \& McClure, 1977) that the colour-magnitude diagram of this cluster shows an unusual colour spread of the giant branch. This triggered a large number of spectroscopic investigations of the cluster giants which indeed found indications for a metallicity spread similar to the case of $\omega$ Cen. Early studies measuring metallicities in a few giants only did not come to conclusive results (Cohen, 1981; Pilachowski et al., 1982). The investigation by Da Costa et al. (2009) on 55 red giants showed a broad range of metallicities between $[\mathrm{Fe} / \mathrm{H}]=-1.9$ and $[\mathrm{Fe} / \mathrm{H}]=-1.45$, in agreement with earlier papers by Lehnert, Bell, \& Cohen (1991) or Norris and Freeman (1983). 
The metallicity spread was later confirmed by Alves-Brito et al. (2012). The works of Marino and collaborators (Marino et al., 2009, 2011) gave a strong indication for the existence of two populations with a difference in $[\mathrm{Fe} / \mathrm{H}]$ of $0.15 \mathrm{dex}$. The two groups differ also in their abundances of s-process elements, the more metal-rich ones showing a higher abundance of s-process-dominated species. This is in agreement with an evolutionary scenario of the cluster where a second generation of stars is enriched in neutron-capture elements by AGB stars (Marino et al., 2011). The split was also found in a sample of M22 subgiants (Marino et al., 2012). While the presence of two populations in M22 or at least a metallicity spread is now widely accepted in the literature, we note that there are also a few papers finding contradicting results, i.e. the existence of only one population (Anthony-Twarog, Twarog, \& Craig, 1995; Monaco et al., 2004; Ivans et al., 2004).

Clement et al. (2001) list $72^{1}$ variable stars known to date in the cluster field. Most of them are RR Lyr stars, but 12 are probably red long-period variables (LPVs), for some of which periods are given. V5, V8, and V9 are marked as cluster members, while for the others the membership is not clear. Recent surveys for variable stars in that cluster include the study by Kaluzny and Thompson (2001) identifying several SX Phe stars and candidate eclipsing binaries. Eight additional variables were identified in the cluster halo by Kravtsov et al. (1994).

While M22 belongs to the most massive globular clusters, a further target of our survey, IC4499, is a sparse cluster of rather low density in the outer halo of the Galaxy. We combine here our results of this cluster with the ones for M22 since both clusters have a similar low mean metallicity. Harris (1996) gives its distance from the sun as $17.6 \mathrm{kpc}$, while Piotto et al. (2002) estimate a reddening value $E(B-V)=0.23$. These values for distance and reddening are in good agreement with results from RR Lyr variables (Storm, 2004). Only a few spectroscopic metallicity determinations are available - see Hankey and Cole (2011) for a recent summary. Values for $[\mathrm{Fe} / \mathrm{H}]$ range between -1.5 and -1.65 (Smith \& Perkin, 1982; Walker \& Nemec, 1996) on the Zinn \& West (1984) scale. There exists also a number of photometric attempts to determine the cluster metallicity which give somewhat lower values around $[\mathrm{Fe} / \mathrm{H}]=-1.75$ (Fusi Pecci et al., 1995; Ferraro et al., 1995). Hankey and Cole (2011) themselves present an extensive spectroscopic study of 43 red giants, finding a metallicity of $[\mathrm{Fe} / \mathrm{H}]=-1.52 \pm$ 0.12 . There is some debate that IC4499 may be $2-4 \mathrm{Gyr}$ younger than the bulk of the metal-poor globular clusters (Ferraro et al., 1995; Salaris \& Weiss, 2002; Walker et al., 2011). IC4499 is famous for its high number of RR Lyr stars (Sarajedini, 1993; Clement et al., 2001; Walker \& Nemec, 1996). However, no LPVs are known in this cluster according to Clement et al. (2001).

\footnotetext{
${ }^{1}$ Including the 2009 update published on their webpage: http://www.astro. utoronto.ca/ cclement/cat/C1833m239.
}

\section{DATA ANALYSIS}

The two clusters are part of our observing programme to identify and characterise LPVs in Galactic globular clusters. The observations of M22 and IC4499 were obtained and analysed the same way as the previous papers in this series (see Section 1). Thus we will give here only a brief summary and refer to Lebzelter and Wood (2005) for details. Our monitoring programme of IC4499 and M22 started in May 2002 with the 50-inch telescope at Mount Stromlo. This telescope was equipped with a two-channel camera used earlier for the MACHO experiment (Alcock et al., 1993). Observations were obtained once to twice a week, but ended unexpectedly after a few months when Mount Stromlo observatory was destroyed by a bush fire. Altogether we collected 21 usable frames for M22 and 27 usable frames for IC4499 over this time span. All observations were done in queue observing mode.

Data from both the blue and the red channels were used for the detection of variables and the determination of light curves. Due to the larger light amplitude of LPVs in the blue than in the red, the blue frames received a higher weight for the detection of variables and the determination of periods. Since the two channels had the same exposure time and since LPVs are typically the brightest stars in the red in an old stellar cluster, several of our variables were over-exposed on the red frames and thus only the blue channel data were useable. Small positional shifts of the cluster on the frame between the observing nights and the presence of an area of bad pixels on the detectors prevented the measurement of all the stars on all our frames.

Variable star detection and extraction of the light curve data was done with the help of the image subtraction code ISIS 2.1 developed by Alard (2000). Stellar fluxes on the reference frames were measured using standard IRAF software. Two images observed in the same night give a differential photometric accuracy for a bright cluster giant around 0.005 mag in the blue. To determine the photometric zero point in $V$, we identified several stars with known $V$ photometry from the survey by Monaco et al. (2004) on the blue frames. No correction has been applied for the difference between Johnson $V$ and MACHO blue. Since the comparison stars were of colour similar to most of our target stars, we expected a rather small difference of around 0.1 magnitudes (Bessell \& Germany, 1999). To calibrate the red MACHO frames, $R$-band magnitudes would have been required. Since these were not available in our case, the red frames were analysed with an arbitrary zero point.

Among the variable stars detected, we selected the LPVs based on the brightness (on the upper giant branch), timescale of the variation (more than $30 \mathrm{~d}$ ), and a total light amplitude in $\mathrm{V}$ of at least $0.03 \mathrm{mag}$. As in the previous papers of this series, Period98 (Sperl, 1998) was used to derive periods from our light curves. Period98 is a code which can compute a discrete Fourier transformation in combination with a least-squares fitting of multiple frequencies on the data. A maximum of two periods was considered for each star. For the period range 
Table 1. Long-period variables in the field of M22.

\begin{tabular}{|c|c|c|c|c|c|c|c|c|c|c|}
\hline Name & RA (2000) & $\operatorname{Dec}(2000)$ & $\begin{array}{l}V_{\text {mean }} \\
{[\mathrm{mag}]}\end{array}$ & $\begin{array}{c}I^{a} \\
{[\mathrm{mag}]}\end{array}$ & $\begin{array}{c}J \\
{[\mathrm{mag}]}\end{array}$ & $\begin{array}{c}H \\
{[\mathrm{mag}]}\end{array}$ & $\begin{array}{c}K \\
{[\mathrm{mag}]}\end{array}$ & $\begin{array}{l}\text { Period } \\
\text { [d] }\end{array}$ & $\begin{array}{c}\Delta V \\
{[\mathrm{mag}]}\end{array}$ & Remark \\
\hline V5 & 183610.57 & -235500.5 & 11.16 & 9.18 & 7.72 & 6.99 & 6.731 & 54 & 0.8 & $\mathrm{P}_{\mathrm{lit}} 93 \mathrm{~d}$ \\
\hline V14 & 183640.52 & -234607.4 & 14.84 & 10.96 & 8.924 & 8.037 & 7.515 & $202^{c}$ & 2.2 & $\begin{array}{l}\text { V1266 Cyg, } \mathrm{P}_{\text {lit }} 202 \mathrm{~d} \\
\text { Likely non-member }\end{array}$ \\
\hline V30 & 183641.05 & -235819.5 & 11.34 & 9.19 & 7.75 & 6.991 & 6.759 & 62 & 0.2 & $\mathrm{P}_{\mathrm{lit}} 82.5 \mathrm{~d}$ \\
\hline $\mathrm{V} 34^{b}$ & 183626.07 & -235533.9 & 12.41 & 9.48 & 8.019 & 7.225 & 6.988 & 63: & 0.2 : & No $P_{\text {lit }}$ \\
\hline V35 & 183624.04 & -235429.3 & 12.03 & 9.41 & 7.948 & 7.171 & 6.949 & 56: & 1.0 & No $P_{1 \text { tit }}$ \\
\hline SLW1 & 183541.00 & -235328.8 & 12.34 & 11.06 & 9.836 & 9.092 & 8.896 & 61 & 2.2 & Likely non-member \\
\hline SLW2 & 183559.09 & -235134.7 & 13.90 & 10.77 & 9.143 & 8.082 & 7.816 & 105 & 1.2 & Likely non-member \\
\hline SLW3 & 183559.44 & -235402.7 & 15.92 & 12.72 & 11.209 & 10.328 & 10.06 & $77:$ & 1.0 & Likely non-member \\
\hline SLW4 & 183617.51 & -235426.3 & 11.41 & 9.32 & 7.82 & 7.011 & 6.783 & 57 & 1.2 & \\
\hline SLW5 & 183618.38 & -235401.3 & 11.15 & 9.62 & 8.289 & 7.53 & 7.306 & 76 & 0.03 & \\
\hline SLW6 & 183619.27 & -235326.7 & 11.18 & 9.53 & 8.013 & 7.688 & 7.257 & 73: & 0.07 & \\
\hline SLW7 & 183621.01 & -235442.5 & 11.28 & 9.68 & 8.407 & 7.614 & 7.438 & 61 & 0.1 & \\
\hline SLW8 & 183621.64 & -235557.0 & 11.90 & 9.36 & 7.933 & 7.163 & 6.929 & 61: & 1.2 & \\
\hline SLW9 & 183625.42 & -235435.6 & 13.23 & 11.60 & 10.512 & 9.878 & 9.689 & 122: & 0.6 & \\
\hline SLW10 & 183626.64 & -234502.9 & 13.71 & 11.51 & 9.432 & 8.498 & 8.104 & $46^{d}$ & 0.03 & \\
\hline SLW11 & 183628.05 & -235323.2 & 11.39 & 9.55 & 8.189 & 7.34 & 7.197 & 79 & 0.07 & Non-member? \\
\hline
\end{tabular}

${ }^{a} I$-band data taken from the DENIS database (The DENIS Consortium, 2005).

${ }^{b}$ Classification as LPV doubtful.

${ }^{c}$ Literature value used. ${ }^{d}$ Long secondary period.

studied here (a few ten days) we do not expect a significant aliasing problem from our sampling of the light curves. Note that, given that the periods of semiregular variables may change from time to time, our results only describe the light variation at the time of observation. This may represent only a small part of a more complex light change. In particular, long time variations on time-scales of a year or more are not accessible with our data set.

\section{RESULTS}

\subsection{M22}

Five known and eleven new LPVs were found in M22. All stars are identified in Table 1, where coordinates, $V$, DENIS $I$, and 2MASS magnitudes, periods, and amplitudes are listed. As in Lebzelter \& Wood (2005) we used the variable-star identifiers given by Clement et al. (2001) where available. All other variables were named SLW with some number. Uncertain periods or amplitudes are marked by a colon. The previously reported LPV candidates V8, V17, V26, V28, and V31 to V33 were outside our studied field of view.

Three of the previously known LPVs in Table 1 have periods listed in the literature which is given in the last column of Table 1. In the case of V14-originally detected by Bailey (1902) — we used the literature period of $202 \mathrm{~d}$, since the length of our time series is not sufficient to cover one complete light cycle. We can confirm that the literature period is in a good agreement with our data in this case. For the other two stars with periods from the literature, however, we find quite different periods. In the case of V5 we note some irregular behaviour at the beginning of our light curve, but the second half clearly supports a period of 54 d, i.e. roughly half the literature value. On the other hand, Wehlau \& SawyerHogg (1977) found a 93-d period to be the most prominent one over a very long time interval. This star appears to show multiple periods.

For V30 the longer period found in the literature seems to be unlikely, although the situation is not as clear as for V5. A reason for the differences in period with former studies for these three stars is unknown. The period might indeed have changed, but it may also be that the time sampling on which the literature values are based was not sufficient since in all cases we get shorter periods. We note that there is a star close to our variable SLW9 that has been reported variable by Kaluzny and Thompson (2001). It is not clear if their star pk6 is different from SLW9. However, Kaluzny and Thompson classified it as an eclipsing binary. We find no obvious indications for binarity in our data, therefore we suppose that Kaluzny and Thompson discovered a different variable.

We further note that the star V9 in this cluster has been previously identified as red variable. Despite the star having colour and brightness similar to other LPVs in our sample, its variability amplitude in $V$ in our data is very small and the star does not show any characteristics of a long-period light change. The period value given in the literature could not be confirmed by our data, although the Wehlau and SawyerHogg (1977) measurements seem to support the variability of this star. We suspect that V9 is currently in a phase of quiescence occasionally found in semiregular variables, and the Wehlau and Sawyer-Hogg data indeed show indications for a variable amplitude.

The light variations of the LPVs in M22 are presented in Figure 1 plotted against time starting with the first date of our time series. They are compared with synthetic light 

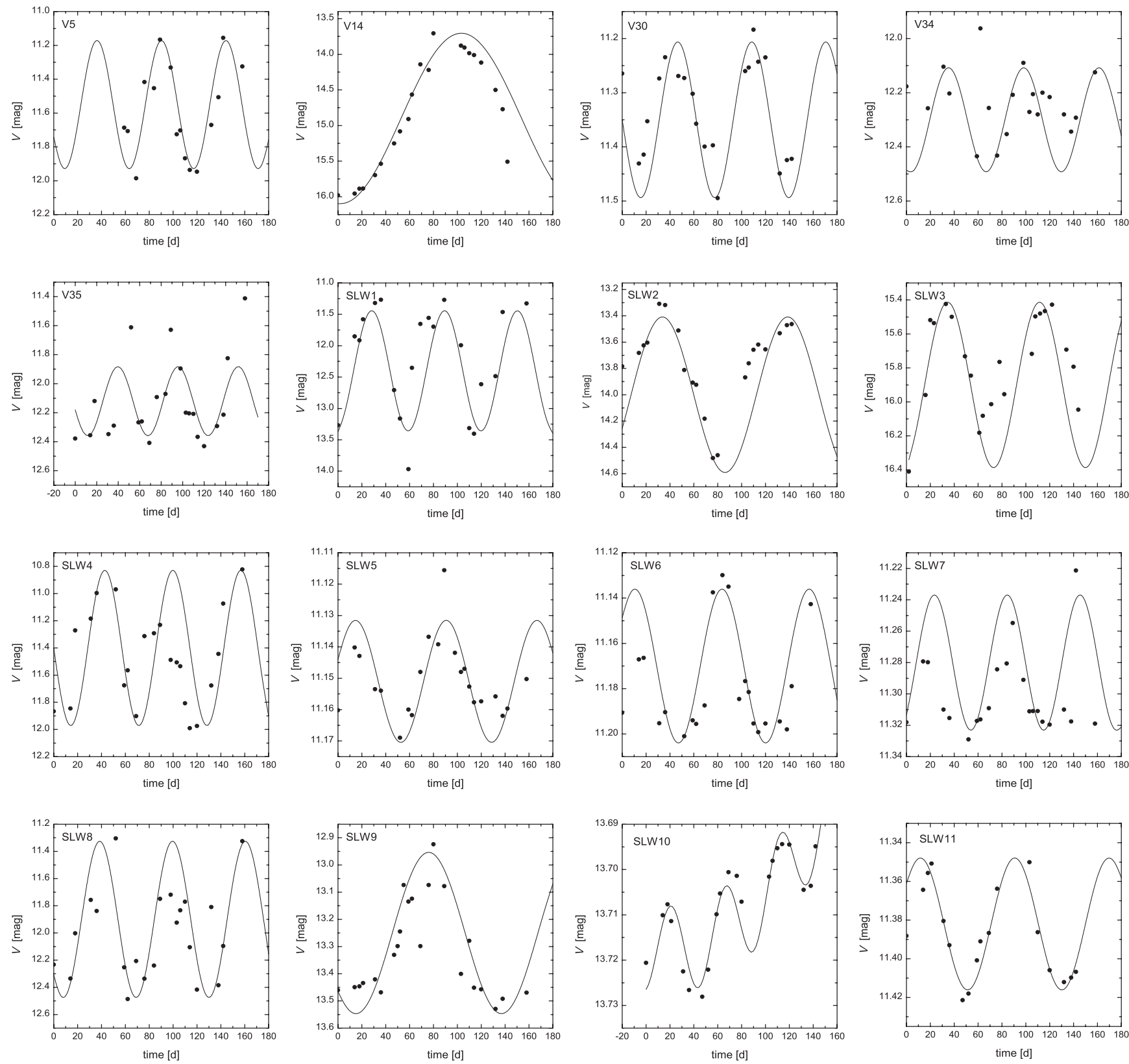

Figure 1. Light change of the long-period variables in M22 plotted against time. Starting date is JD 2452420.

curves based on the period given in Table 1. Photometry data are given in Table 4 . SLW10 shows a very long period variation in addition to the 46-d period. Such long periods are a well-known phenomenon in red variables (Percy et al., 2003; Kiss et al., 1999), but its nature is not understood yet (Wood, Olivier, \& Kawaler, 2004). For illustrative purposes, a long time trend has been added also to the synthetic light curve. SLW10 is also interesting since it is possibly identical with an X-ray source.

It is quite obvious that most stars show some kind of irregular behaviour on top of the adopted periodic light change as expected for semiregular variables. We note that the period of SLW9, although it seems to give a nice fit, is classified as uncertain since we could not detect a second maximum. The light curve of V34 is very unusual for an LPV. Plotted against phase it rather resembles the one expected for an eclipsing binary. We therefore suspect that for this star the observed variability is caused by a companion.

In addition to the variables for which we could estimate a period, we list in Table 2 several red stars that show variability but without a clear periodicity. This may be due to a truly irregular nature of their light change or a larger photometric error, e.g. if there is another star nearby. We did not investigate these cases further.

A proper motion study for individual stars in NGC 6656 can be found in the paper by Zloczewski et al. (2012). 
Table 2. Long-period-variable candidates of M22 with unknown periods.

\begin{tabular}{lcccrcrr}
\hline \hline Name & RA (2000) & Dec (2000) & $\begin{array}{c}V_{\text {mean }} \\
{[\mathrm{mag}]}\end{array}$ & $\begin{array}{r}I^{a} \\
{[\mathrm{mag}]}\end{array}$ & $\begin{array}{c}J \\
{[\mathrm{mag}]}\end{array}$ & $\begin{array}{c}H \\
{[\mathrm{mag}]}\end{array}$ & $\begin{array}{c}K \\
{[\mathrm{mag}]}\end{array}$ \\
\hline V9 $^{b}$ & 183608.19 & -235502.8 & 11.06 & 9.34 & 7.701 & 6.926 & 6.676 \\
SLW12 & 183602.18 & -235650.6 & 12.35 & 10.57 & 9.427 & 8.755 & 8.583 \\
SLW13 & 183610.22 & -234844.4 & 11.94 & 9.35 & 7.842 & 7.005 & 6.737 \\
SLW14 & 183614.57 & -235245.4 & 13.40 & 12.36 & 11.37 & 10.794 & 10.708 \\
SLW15 & 183620.64 & -235136.0 & 12.89 & 10.01 & 8.822 & 8.072 & 7.868 \\
SLW16 & 183623.47 & -235453.8 & 11.23 & 9.40 & 7.988 & 7.23 & 6.971 \\
SLW17 & 183626.13 & -235451.0 & 11.32 & 10.04 & 8.752 & 8.013 & 7.881 \\
SLW18 & 183626.25 & -235232.0 & 12.79 & 11.26 & 10.161 & 9.491 & 9.352 \\
SLW19 & 183626.27 & -235148.3 & 12.62 & 10.82 & 9.782 & 9.136 & 9.011 \\
SLW20 & 183630.53 & -235357.8 & 11.59 & 9.92 & 8.697 & 8.007 & 7.827 \\
SLW21 & 183633.01 & -235435.6 & 11.28 & 10.09 & 9.114 & 8.589 & 8.401 \\
SLW22 & 183633.01 & -234244.1 & 14.15 & & 8.719 & 7.817 & 7.469 \\
\hline \hline
\end{tabular}

${ }^{a} I$-band data taken from the DENIS database (The DENIS Consortium, 2005).

${ }^{b} \mathrm{P}_{\text {lit }} 87.7 \mathrm{~d}$.

Table 3. Long-period variables of IC4499.

\begin{tabular}{lcccccccrr}
\hline \hline Name & RA (2000) & Dec (2000) & $\begin{array}{c}V_{\text {mean }} \\
{[\mathrm{mag}]}\end{array}$ & $\begin{array}{c}I^{a} \\
{[\mathrm{mag}]}\end{array}$ & $\begin{array}{c}J \\
{[\mathrm{mag}]}\end{array}$ & $\begin{array}{c}H \\
{[\mathrm{mag}]}\end{array}$ & $\begin{array}{c}K \\
{[\mathrm{mag}]}\end{array}$ & $\begin{array}{c}\text { Period } \\
{[\mathrm{d}]}\end{array}$ & $\begin{array}{c}\Delta V \\
{[\mathrm{mag}]}\end{array}$ \\
\hline SLW1 & 150022.74 & -821235.9 & 12.19 & 11.19 & 10.203 & 9.587 & 9.424 & 67 & 0.8 \\
SLW2 & 150120.37 & -821243.5 & 13.44 & 11.71 & 10.805 & 10.183 & 10.027 & 35 & 0.8 \\
\hline \hline
\end{tabular}

${ }^{a} I$-band data taken from the DENIS database (The DENIS Consortium, 2005).

According to these authors, V9, V34, and SLW6 are likely cluster members, V30, SLW11, and SLW20 are probably non-members. However, V30 is listed as a member based on its radial velocity measured by Peterson and Cudworth (1994). These authors find a mean radial velocity of approximately $147.6 \pm 9.8 \mathrm{~km} / \mathrm{s}$ for a sample of 170 objects they consider cluster members. Their list gives also support to the cluster membership of V5, V9, and SLW4. We note that the LPV candidate V8 listed by Clement et al. (2001), which was not observed within our programme, fulfills both membership criteria as well.

From the 2MASS $J$ and $K$ photometry, we find that V5, V9, V30, V34, V35, SLW1, SLW4, SLW5, SLW7, SLW8, SLW9, and SLW11 are located on the cluster's giant branch (see Figure 2). SLW1 and SLW9 are more than 2 mag below the tip of the giant branch and are thus likely red-giant-branch (RGB) stars. It is unlikely that stars with their relatively large amplitudes would be found this far below the RGB tip, so they are likely to be non-members. SLW6 is offset to the blue in $(J-K)$, but its $(V-I)$ colour is similar to the other red giants. Since it is probably a cluster member due to its proper motion (Zloczewski et al., 2012), we suspect that the 2MASS $K$ brightness of this star may be incorrect. V14, SLW2, SLW3, and SLW10 are found on the right side of the giant branch. It is difficult to decide on their cluster membership since their colour and brightness may be affected by circumstellar dust or their variability (in particular for the large amplitude variable V14). However, they may also be background sources.

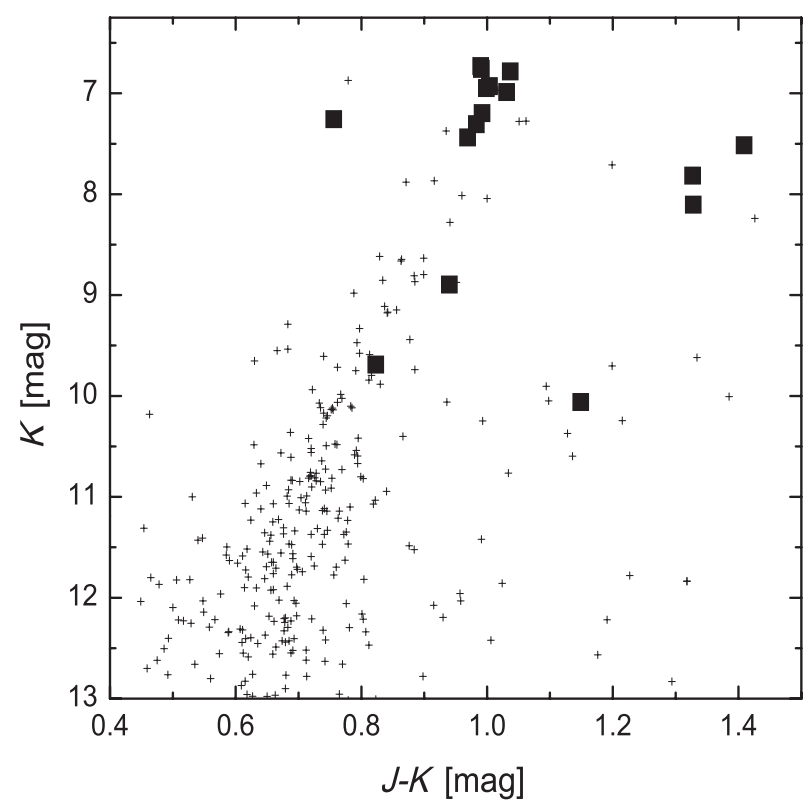

Figure 2. Colour-magnitude diagram of the central part of M22 using 2MASS $J$ and $K$ photometry. The filled boxes mark the variables from Table 1.

\subsection{IC4499}

In the MACHO data of the second cluster, two objects were found to have significant variability, both previously unreported. They are listed in Table 3 and their light curves 

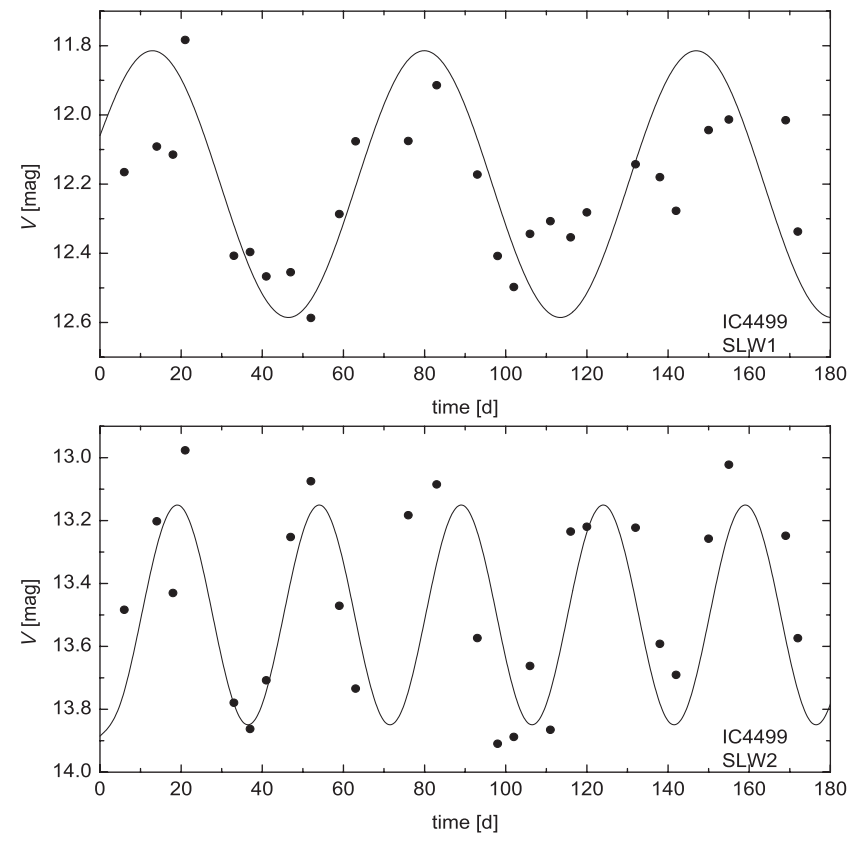

Figure 3. Light change of the two newly detected variables in IC4499 plotted against time. Starting date is JD 2452420.

plotted against time are presented in Figure 3. Photometry data for both stars can be found in Table 5. No long secondary period has been found in any one of them, both of them are monoperiodic.

No proper motion data have been reported in literature for IC4499. Hankey and Cole (2011) published a radial velocity study of stars in that field, but none of our LPV candidates was observed. From their $K$ brightness, both variables are found at the top of the RGB, and they are within a 5-arcmin radius of the cluster centre. However, their $J-K$ colour is slightly too blue compared with the bulk of probable RGB stars in IC4499 (Hankey \& Cole, 2011). SLW1 is also too blue in $V-I$ for an RGB star according to the photometry presented by Walker and Nemec (1996), while SLW2 has an appropriate $V-I$ value. For both stars, their brightness in $V$ places them above the cluster's giant branch. Their membership is therefore not clear, and a possible coincidence of their location close to the cluster centre has to be counterbalanced with their too blue colour. As Hankey and Cole noted, the cluster is seen through the outer parts of the Galactic bulge, therefore it cannot be excluded that these objects are actually bulge stars. Due to their brightness and colour we tend to classify both objects as non-members.

\section{DISCUSSION}

For both clusters studied here, the fields observed will likely contain a considerable contribution from field (or bulge) stars. To discuss the LPVs in M22 and IC4499 we thus have to distinguish cluster members and field stars first. As shown above, velocity and proper motion data found in the literature

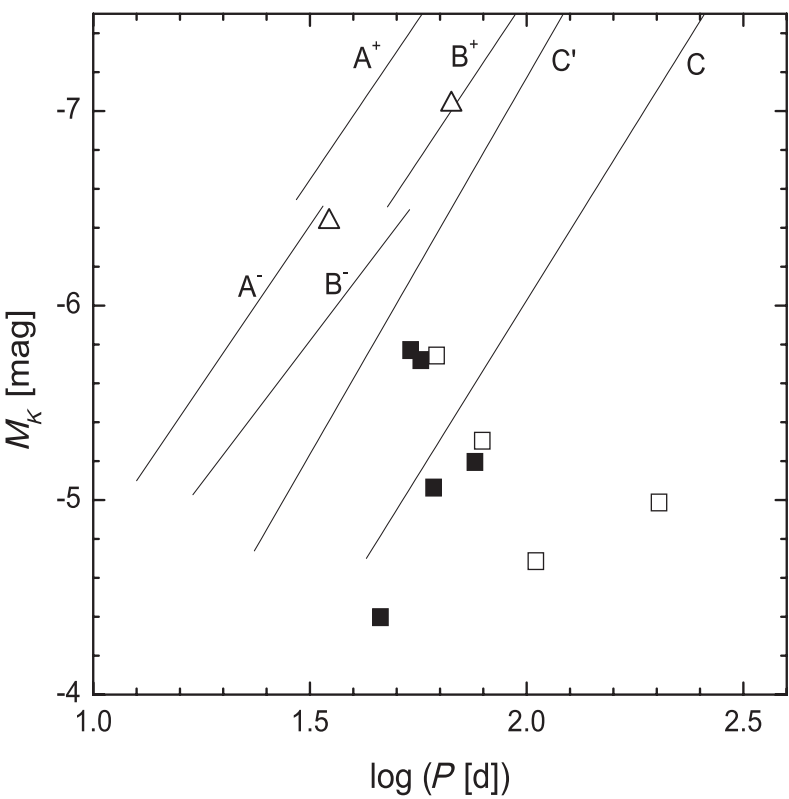

Figure 4. $\log P-K$ diagram of the variables found in this study. Boxes denote stars in M22 while the two objects in IC4499 are marked with triangles. Open symbols indicate uncertain membership. Only stars with reliable periods based on our data are plotted. Furthermore, the plot gives the location of the $\log P-K$ relations for LPVs derived from the Large Magellanic Cloud by Ita et al. (2004).

do not allow a clear separation in all cases. We decided to do a first selection of members on the basis of their location in the 2MASS colour-magnitude diagrams (Figure 2 for M22; see Hankey and Cole (2011) for IC4499). As a next step, we did a cross-check of these candidates using their pulsational properties.

In Figure 4 we show a $\log P-M_{K}$ diagram for the variables listed in Tables 1 and 3 with reliable periods. Absolute magnitudes have been calculated using a distance modulus of 12.46 and 16.23 for M22 and IC4499, respectively. Reddening values $E(B-V)$ of 0.38 and 0.23 have been used. Interstellar extinction in $K$ has been calculated using the relation given in Cardelli, Clayton, \& Mathis (1989). We also added the $\log P-K$ relations for the large magellanic clouds (LMC) given by Ita et al. (2004) shifted with a distance modulus of 18.5 and transformed from the Las Campanas Observatory (LCO) to the 2MASS system following Carpenter (2001). Variables with uncertain cluster membership are marked by open symbols.

Based on the $\log P-K$ diagram shown in Figure 4 we further exclude SLW1 ${ }^{2}$, SLW2, and the large amplitude variable V14. Since the period is also used as a selection criterion now, we remove all variables with unknown or uncertain period as well. We note that the period from Wehlau and Sawyer-Hogg (1977) would place the star V9 (Table 2) close to the other M22 variables in this diagram. Our resulting sample includes seven stars, namely V5, V30, SLW4, SLW5, SLW7, SLW10, and SLW11. The membership of SLW11 and

\footnotetext{
${ }^{2}$ Not visible in Figure 4 due to its low $K$ brightness.
} 
Table 4. Visual magnitudes of the long-period variables in the field of NGC 6656.

\begin{tabular}{|c|c|c|c|c|c|c|c|c|}
\hline Julian date & $\begin{array}{c}\text { V5 } \\
{[\mathrm{mag}]}\end{array}$ & $\begin{array}{c}\mathrm{V} 14 \\
{[\mathrm{mag}]}\end{array}$ & $\begin{array}{c}\mathrm{V} 30 \\
{[\mathrm{mag}]}\end{array}$ & $\begin{array}{c}\mathrm{V} 34 \\
{[\mathrm{mag}]}\end{array}$ & $\begin{array}{c}\text { V35 } \\
{[\mathrm{mag}]}\end{array}$ & $\begin{array}{l}\text { SLW1 } \\
{[\mathrm{mag}]}\end{array}$ & $\begin{array}{l}\text { SLW2 } \\
{[\mathrm{mag}]}\end{array}$ & $\begin{array}{l}\text { SLW3 } \\
\text { [mag] }\end{array}$ \\
\hline 2452420 & 11.959 & 15.980 & 11.265 & 12.177 & 12.378 & 13.270 & 13.784 & 16.410 \\
\hline 2452434 & 12.000 & 15.957 & 11.431 & 12.731 & 12.356 & 11.853 & 13.683 & 15.960 \\
\hline 2452438 & 11.439 & 15.887 & 11.414 & 12.258 & 12.120 & 11.915 & 13.625 & 15.519 \\
\hline 2452451 & 11.709 & 15.886 & 11.353 & 12.104 & 12.349 & 11.580 & 13.604 & 15.537 \\
\hline 2452456 & 11.825 & 15.698 & 11.274 & 12.203 & 12.289 & 11.320 & 13.309 & 15.424 \\
\hline 2452472 & 11.054 & 15.538 & 11.235 & 11.196 & 11.612 & 11.268 & 13.319 & 15.499 \\
\hline 2452479 & 11.687 & 15.253 & 11.269 & 12.435 & 12.267 & 12.708 & 13.512 & 15.732 \\
\hline 2452482 & 11.707 & 15.084 & 11.273 & 11.963 & 12.261 & 13.162 & 13.812 & 15.846 \\
\hline 2452489 & 11.986 & 14.911 & 11.302 & 12.257 & 12.409 & 13.969 & 13.908 & 16.182 \\
\hline 2452496 & 11.417 & 14.567 & 11.357 & 12.433 & 12.093 & 12.354 & 13.925 & 16.082 \\
\hline 2452504 & 11.453 & 14.142 & 11.399 & 12.353 & 12.071 & 11.653 & 14.182 & 16.014 \\
\hline 2452509 & 11.166 & 14.221 & 11.397 & 12.208 & 11.629 & 11.559 & 14.482 & 15.765 \\
\hline 2452518 & 11.331 & 13.708 & 11.495 & 12.090 & 11.896 & 11.697 & 14.459 & 15.955 \\
\hline 2452523 & 11.726 & 13.880 & 11.260 & 12.271 & 12.200 & 11.271 & 13.868 & 15.717 \\
\hline 2452526 & 11.703 & 13.906 & 11.253 & 12.206 & 12.205 & 11.9 & 61 & 15.497 \\
\hline 2452530 & 11.868 & 13.985 & 11.184 & 12.280 & 12.209 & 13. & & 15.480 \\
\hline 2452534 & 11.936 & 14.012 & 11.243 & 12.200 & 12.367 & 13.405 & 19 & 15.465 \\
\hline 2452540 & 11.947 & 14.117 & 11.235 & 12.216 & 12.432 & 12.616 & 13.655 & 15.428 \\
\hline 2452552 & 11.671 & 14.503 & 11.449 & 12.280 & 12.293 & 12.487 & 13.533 & 15.693 \\
\hline 2452558 & 11.507 & 14.773 & 11.425 & 12.344 & 12.214 & 11.465 & 13.471 & 15.794 \\
\hline 2452562 & 11.156 & 15.509 & 11.422 & 12.292 & 11.825 & 11.402 & 13.463 & 16.046 \\
\hline \multirow[t]{2}{*}{2452578} & 11.325 & 15.537 & 11.398 & 12.125 & 11.412 & 11.326 & 13.492 & 16.326 \\
\hline & SLW4 & SLW5 & SLW6 & SLW7 & SLW8 & SLW9 & SLW10 & $\overline{\text { SLW11 }}$ \\
\hline Julian Date & {$[\mathrm{mag}]$} & {$[\mathrm{mag}]$} & {$[\mathrm{mag}]$} & {$[\mathrm{mag}]$} & {$[\mathrm{mag}]$} & [mag] & {$[\mathrm{mag}]$} & [mag] \\
\hline 2452420 & 11.867 & 11.160 & 11.191 & 11.318 & 12.234 & 13.461 & 13.721 & 11.388 \\
\hline 2452434 & 11.846 & 11.140 & 11.167 & 11.279 & 12.336 & 13.449 & 13.710 & 11.364 \\
\hline 2452438 & 11.271 & 11.143 & 11.166 & 11.280 & 12.003 & 13.447 & 13.708 & 11.356 \\
\hline 2452441 & 11.185 & 11.153 & 11.1 & 11.310 & 11.757 & & & 11.351 \\
\hline 2452451 & 10.997 & 11.154 & 11.190 & 11.3 & 11.8 & & & 11.380 \\
\hline 2452456 & 10.970 & 11.169 & 11.201 & 11.329 & 11.305 & 13.468 & 13.727 & 11.393 \\
\hline 2452467 & 11.676 & 11.160 & 11.194 & 11.317 & 12.254 & 13.331 & 13.728 & 11.421 \\
\hline 2452472 & 11.565 & 11.162 & 11.196 & 11.316 & 12.487 & 13.245 & 13.722 & 11.418 \\
\hline 2452479 & 11.902 & 11.148 & 11.187 & 11.309 & 12.207 & 13.135 & 13.710 & 11.401 \\
\hline 2452482 & 11.313 & 11.137 & 11.138 & 11.284 & 12.337 & 13.124 & 13.705 & 11.391 \\
\hline 2452489 & 11.293 & 11.139 & 11.130 & 11.281 & 12.240 & 13.299 & 13.701 & 11.387 \\
\hline 2452496 & 11.231 & 11.116 & 11.135 & 11.259 & 11.749 & 13.074 & 13.701 & 11.364 \\
\hline 2452500 & 11.488 & 11.142 & 11.185 & 11.291 & 11.719 & 12.924 & 13.707 & 11.323 \\
\hline 2452523 & 11.506 & 11.148 & 11.177 & 11.311 & 11.925 & 13.078 & 13.702 & 11.350 \\
\hline 2452526 & 11.535 & & & & & & & \\
\hline 2452530 & 11.807 & & & & 11.770 & 13.279 & 13.695 & 11.386 \\
\hline 2452534 & 11.991 & 11.158 & 11.199 & 11.318 & 12.106 & 13.451 & 13.694 & \\
\hline 2452540 & 11.975 & 11.157 & 11.195 & 11.320 & 12.418 & 13.458 & 13.694 & 11.406 \\
\hline 2452552 & 11.677 & 11.156 & 11.194 & 11.310 & 11.810 & 13.529 & 13.704 & 11.412 \\
\hline 2452558 & 11.445 & 11.162 & 11.198 & 11.318 & 12.385 & 13.493 & 13.704 & 11.410 \\
\hline 2452562 & 11.074 & 11.160 & 11.179 & 11.221 & 12.096 & 13.481 & 13.695 & 11.407 \\
\hline 2452578 & 10.823 & 11.150 & 11.143 & 11.319 & 11.325 & 13.470 & 13.690 & \\
\hline
\end{tabular}

V30 is somewhat uncertain according to the literature data, but we decided to leave them in the sample, too. From their brightness, the stars are either on the RGB or on the AGB. In our second cluster, IC4499, the membership of both LPV candidates is doubtful due to their brightness and colour.

Among the clusters studied so far within our monitoring program, M22 and IC4499 are the two with the lowest metallicity. Nevertheless, LPVs are found at least in one of them, M22. Among our seven good candidates, four have $V$ amplitudes of a few tenth of a magnitude or more. We remind the reader that a mira-like variability with a $V$ amplitude of several magnitudes is directly related to the high temperature sensitivity of the TiO bands in the visual part of the spectrum and therefore not expected for low metallicity stars like the ones we study here. This is in agreement with the typical amplitudes found in LPVs in the somewhat more metal-rich clusters NGC 362 and NGC 2808 (Lebzelter \& Wood, 2011). Considering the detection of LPVs in the even more metalpoor cluster M15 by McDonald et al. (2010) we propose that this kind of variability appears at the upper end of the giant branch also at much lower metallicity than expected previously (Frogel \& Elias, 1988). 
Table 5. Visual magnitudes of the long-period variables of IC4499.

\begin{tabular}{lll}
\hline \hline Julian date & $\begin{array}{l}\text { SLW1 } \\
{[\mathrm{mag}]}\end{array}$ & $\begin{array}{l}\text { SLW2 } \\
{[\mathrm{mag}]}\end{array}$ \\
\hline 2452426 & 12.165 & 13.484 \\
2452434 & 12.091 & 13.202 \\
2452438 & 12.115 & 13.430 \\
2452441 & 11.783 & 12.976 \\
2452453 & 12.407 & 13.779 \\
2452457 & 12.396 & 13.863 \\
2452461 & 12.467 & 13.708 \\
2452467 & 12.455 & 13.252 \\
2452472 & 12.587 & 13.075 \\
2452479 & 12.287 & 13.471 \\
2452483 & 12.076 & 13.734 \\
2452496 & 12.075 & 13.183 \\
2452503 & 11.914 & 13.085 \\
2452513 & 12.172 & 13.574 \\
2452518 & 12.408 & 13.909 \\
2452522 & 12.498 & 13.888 \\
2452526 & 12.344 & 13.662 \\
2452531 & 12.307 & 13.865 \\
2452536 & 12.354 & 13.235 \\
2452540 & 12.282 & 13.220 \\
2452552 & 12.143 & 13.223 \\
2452558 & 12.180 & 13.592 \\
2452562 & 12.277 & 13.690 \\
2452570 & 12.044 & 13.258 \\
2452575 & 12.013 & 13.022 \\
2452589 & 12.015 & 13.242 \\
2452592 & 12.337 & 13.574 \\
\hline \hline
\end{tabular}

Beside $\mathrm{TiO}$ playing less of a role for the visual amplitude, in low metallicity clusters, the LPVs are found at a lower luminosity compared to, e.g. the LPVs in 47 Tuc. This trend seen already in NGC 362 and NGC 2808 is clearly continued in M22 with the most luminous cluster LPV having $M_{K}$ below -6 , more than 1 mag below the most luminous LPVs in 47 Tuc. At the same time, the LPV phenomenon sets in at a lower brightness than in 47 Tuc. Should the two variables found in the field of IC4499 be indeed cluster members, this would naturally contradict the findings from the other clusters. It could be explained if this cluster is indeed younger than clusters like M22, but this option has been rejected in the most recent study of this issue by Walker et al. (2011). Therefore, it would be important to clarify their cluster membership or non-membership unambiguously. That we did not find any LPV candidates in IC4499 at a lower luminosity is probably due to the cluster's distance and significantly smaller size in terms of member stars.

Finally, we take a look at the location of the seven M22 LPVs relative to the LPV $\log$ P- $K$ relations found for the LMC (Figure 4). We find four stars close to sequence C, which has been related to fundamental mode pulsation, and three stars between sequence $\mathrm{C}$ and C'. The LPV candidate V8 from Clement et al. (2001) would be placed close to sequence $\mathrm{C}$ as well using its literature period $(61 \mathrm{~d})$ and 2MASS $K$-magnitude. However, that star is slightly shifted to the blue in $J-K$ relative to the cluster's giant branch, so that its classification as LPV is not totally clear.

Our variables seem to be somewhat offset from the sequences - shifted either to a longer period or a lower brightness. From our previous work (Lebzelter \& Wood, 2011, in particular) we know that both low metallicity and mass loss tend to shift the $\log P-K$ relations to the right in such diagrams, in agreement with the very low metallicity of the M22 stars. The alternative explanation would be a small uncertainty in the distance to M22, which seems more unlikely. An interesting feature of the diagram is that the most luminous LPVs in M22 are not found on sequence $\mathrm{C}$ but on sequence $C^{\prime}$. The low number of variables with reliable data prohibits the drawing of further conclusions on this finding.

To sum up, we report the detection of 24 previously unknown LPVs in the fields of the two metal-poor globular clusters M22 and IC4499. Periods were derived for 17 of the LPVs and cluster membership was discussed. The detection of LPVs as members of these metal-poor clusters adds to the sketching of a complete picture of variability on the upper giant branch at various metallicities.

\section{ACKNOWLEDGEMENTS}

TL acknowledges support by the Austrian Science Fund under project number P23737-N16. The authors wish to thank Warren Hankey for providing radial velocity data for IC4499.

\section{References}

Alard, C. 2000, A\&A, 144, 363

Alcock, C., Allsman, R. A., Axelrod, T. S., et al. 1993, in: Sky Surveys.Protostars to Galaxies, ed. Soifer, B.T., ASP Conference Series vol. 43, 291. San Francisco.

Alves-Brito, A., Yong, D., Melendez, J., Vasquez, S., \& Karakas, A. I. 2012, A\&A, 540, A3

Anthony-Twarog, B. J., Twarog, B. A., \& Craig, J. 1995, ASP, 107, 32

Bailey, S. I. 1902, AnHar, 38, 1B

Bessell, M. S., \& Germany, L. M. 1999, PASP, 111, 1421

Cardelli, J. A., Clayton, G. C., \& Mathis, J. S. 1989, ApJ, 345, 245

Carpenter, J. M. 2001, AJ, 121, 2851

Clement, C. M., Muzzin, A., Dufton, Q., et al. 2001, ApJ, 122, 2587

Cohen, J. G. 1981, ApJ, 247, 869

Da Costa, G. S., Held, E. V., Saviane, I., \& Gullieuszik, M. 2009, ApJ, 705, 1481

The DENIS Consortium 2005, 3rd release of the DENIS database, CDS/ADC Collection of Electronic Catalogues, 2263

Ferraro, F. R., Fusi Pecci, F., Testa, V., Greggio, L., Corsi, C. E., Buonanno, R., Terndrup, D. M., Zinnecker, H. 1995, MNRAS, 275,1057

Frogel, J. A., \& Elias, J. H. 1988, ApJ, 324, 823

Fusi Pecci, F., Bellazzini, M., Cacciari, C., \& Ferraro, F. R. 1995, AJ, 110, 1664

Hankey, W.J., \& Cole, A. A. 2011, MNRAS 411, 1536

Harris, W. E. 1996, AJ, 112, 1487

Hesser, J. E., Hartwick, F. D. A., \& McClure, R. D. 1977, ApJ, 33, 471

Ita, Y., Tanabe, T., Matsunaga, N., et al. 2004, MNRAS, 347, 720 
Ivans, I. I., Sneden, C., Wallerstein, G., Kraft, R. P., Norris, J. E., Fulbright, J. P., Gonzalez, G. 2004, MemSAI, 75, 286

Kaluzny, J., \& Thompson, I. B. 2001, A\&A, 373, 899

Kamath, D., Wood, P. R., Soszynski, I., \& Lebzelter, T. 2010, MNRAS, 408, 522

Kiss, L. L., Szatmáry, K., Cadmus, R. R., Jr., Mattei, J. A. 1999, A\&A, 346, 542

Kravtsov, V. V., Samus, N. N., Alcaino, G., \& Liller, W. 1994, AstL, 20, 339

Kunder, A., Stetson, P. B., Cassisi, S., et al. 2013, AJ, 146, 119

Lebzelter, T., Nowotny, W., Höfner, S., Lederer, M. T., Hinkle, K. H., Aringer, B. 2010, A\&A, 517, A6

Lebzelter, T., \& Wood, P. R. 2005, A\&A, 441, 1117

Lebzelter, T., \& Wood, P. R. 2007, A\&A, 475, 643

Lebzelter, T., \& Wood, P. R. 2011, A\&A, 529, A137

Lehnert, M. D., Bell, R. A., \& Cohen, J. G. 1991, ApJ, 367, 514

Marino, A. F., Milone, A. P., Piotto, G., Villanova, S., Bedin, L. R., Bellini, A., Renzini, A. 2009, A\&A, 505, 1099

Marino, A. F., Milone, A. P., Sneden, C., et al. 2012, A\&A, 541, A15

Marino, A. F., Sneden, C., Kraft, R. P., et al. 2011, ApJ, 532, A8

McDonald, I., van Loon, J., Dupree, A., \& Boyer, M. 2010, MNRAS, 405, 1711

Monaco, L., Pancino, E., Ferraro, F. R., \& Bellazzini, M. 2004, MNRAS, 349, 1278
Norris, J., \& Freeman, K. C. 1983, ApJ, 266, 130

Percy, J. R., Besla, G., Velocci, V., \& Henry, G. W. 2003, PASP, 115,479

Peterson, R. C., \& Cudworth, K. C. 1994, ApJ, 420, 612

Pilachowski, C., Leep, E. M., Wallerstein, G., \& Peterson, R. C. 1982, ApJ, 263, 187

Piotto, G., King, I. R., Djorgovski, S. G., et al. 2002, A\&A 391, 945

Salaris, M., \& Weiss, A. 2002, A\&A, 388, 492

Sarajedini, A. 1993, AJ, 105, 2172

Smith, H. A., \& Perkin, G. J. 1982, ApJ, 261, 576

Sperl, M. 1998, CoAst, 111, 1

Storm, J. 2004, A\&A, 415, 987

van den Berg, D. A., Brogaard, K., Leaman, R., \& Casagrande, L. 2013, ApJ, 775, 134

Walker, A. R., Kunder, A. M., Andreuzzi, G., et al. 2011, MNRAS, 415,643

Walker, A. R., \& Nemec, J. M. 1996, AJ, 112, 2020

Wehlau, A., \& Sawyer-Hogg, H. 1977, AJ, 82, 137

Wood, P. R., Olivier, A. E., \& Kawaler, S. D. 2004, in: Variable stars in the local group, eds. D.W. Kurtz and K. Pollards,ASP Conference Series, vol. 310, 322. San Francisco

Zinn, R., \& West, M. J. 1984, ApJS, 55, 45

Zloczewski, K., Kaluzny, J., Rozyczka, M., Krzeminski, W., \& Mazur, B. 2012, AcA, 62, 357 